\title{
Low-cost Miniaturization of Gel Document System Using Blue LED
}

\author{
Seul-Bit-Na Koo, ${ }^{1,2}$ Dong-Geon Jo, ${ }^{2,3}$ Chan-Young Park, ${ }^{1,2}$ \\ Yu-Seop Kim, ${ }^{1,2}$ Hye-Jeong Song, ${ }^{1,2}$ and Jong-Dae Kim ${ }^{1,2 *}$ \\ ${ }^{1}$ Department of Convergence Software, Hallym University, \\ 1 Hallymdaehak-gil, Chuncheon-si, Gangwon-do 24252, Korea \\ ${ }^{2}$ Bio-IT Research Center, Hallym University, \\ 1 Hallymdaehak-gil, Chuncheon-si, Gangwon-do 24252, Korea \\ ${ }^{3}$ Department of Electronics Engineering, Hallym University, \\ 1 Hallymdaehak-gil, Chuncheon-si, Gangwon-do 24252, Korea \\ (Received May 27, 2018; accepted October 11, 2018)
}

Keywords: gel document system, blue LED, open platform, smartphone camera

In this paper, we propose an inexpensive and compact gel document system in an Internet-ofThings (IoT) form employing recent open platforms. The proposed system adopts a smartphone camera developed for an open platform and uses blue LEDs as the lighting device with a recently commercialized light diffuser. The camera is controlled with the open IoT platform to implement the system in the IoT form. Employing open platforms greatly reduces the cost and size of the gel document system. The light diffusers are experimentally compared and the diffuser that made the proposed system more compact is selected. The proposed system has good characteristics of the IoT system such as low price, small installation space, and Internet controllability.

\section{Introduction}

Nucleic acid electrophoresis is an analytical technique used to isolate DNA or RNA fragments in terms of size and reactivity. It has become one of the most important technologies in modern life science and has been widely used in analytical techniques in a variety of fields such as molecular biology, genetics, microbiology, biochemistry, forensics, food science, and botany. ${ }^{(1-8)}$ The electrophoresis gel is visualized through a gel document system consisting of a UV or blue light transilluminator and a camera..$^{(1,3,4,8,9)}$ It is desirable to use a blue light transilluminator if possible, since UV light is not only harmful to the experimenter but also causes DNA damage during analysis. ${ }^{(4,10-14)}$

Generally, a gel document system is usually large because it includes UV fluorescence tubes, a darkroom, a high-performance camera for image capture, and a personal computer for camera control. ${ }^{(15)}$ Even with the blue transilluminator, there is still room for further reduction in size because of the height required to achieve uniform light. This occupies much space in the

*Corresponding author: e-mail: kimjd@hallym.ac.kr https://doi.org/10.18494/SAM.2019.2088 
laboratory, causing inconvenience to the user. Therefore, we propose a low-cost miniaturized gel document system that solves space constraints by constructing the system in an Internetof-Things (IoT) form and employing open platforms. We adapted a blue transilluminator, which is harmless to the human body and DNA, as mentioned above. The blue LEDs of the transilluminator are semipermanent with an average lifetime of 50000 hours and much cheaper and smaller than UV fluorescence tubes. Therefore, they also contribute to not only the miniaturization of the gel document system but also cost reduction.

Until now, the digital single lens reflex (DSLR) camera has been the most cost-effective imaging device for the gel documentation system. Web cams have been applied to reduce the cost at the sacrifice of performance. ${ }^{(15)}$ However, cameras for smartphones have image quality that has been rapidly improving owing to the rapid market growth, and are being continuously released targeting open platform computers. As they usually are very compact and use optics with small focal lengths, it is easy to create compact systems with them. Therefore, adopting an open platform camera instead of a DSLR camera not only leads to low cost and high-quality gel images but also makes it easy to miniaturize the system by reducing the shooting distance.

The diffuser, which is an integral part of the transilluminator, is also improving rapidly in performance and cost owing to recent advances in LED display and lighting technology. Smart devices that require more compact illumination tend to use LEDs with freeform lenses instead of diffusers for homogeneous illumination, but it is not appropriate to apply them to bioinstruments with a relatively small market size.

The next generation of computing is beyond the limits of traditional desktop computers. Many sensing systems surrounding us in the IoT paradigm are embedded in the environment invisibly in the form of a network. ${ }^{(16-18)}$ As part of this trend, bio-instruments will also be integrated into the environment and connected to the Internet. It is therefore desirable to be able to reduce the size of the system and remotely control and monitor the system over the Internet. The data generated by the bio-instruments will ultimately be linked and analyzed in the cloud database. Visualization of data or analytical results is also performed on various smart devices connected to the Internet. When a detection system is developed in the IoT format, it is important to analyze and design a very different user interface from the existing desktop environment. ${ }^{(19)}$ On the other hand, the open platform makes it easy to implement the system in the IoT formmat, enabling the control and monitoring of the system anytime, anywhere. Thus, by using an open platform and camera, we can implement the gel document system in a compact, low-cost IoT format. ${ }^{(3)}$ In this work, we try to implement the gel document system in the IoT form to meet the trend of the latest technology. A low-cost and compact system comprising an open platform single-board computer and a smartphone camera for open platforms is proposed. A method of selecting a diffuser for homogeneous illumination at the smallest height, which is mandatory for compactness, is also presented.

\section{Materials and Methods}

Figure 1 shows the overall architecture of the proposed gel document system. The system consists of a dark room, a camera, and a transilluminator, as in typical gel document systems. 


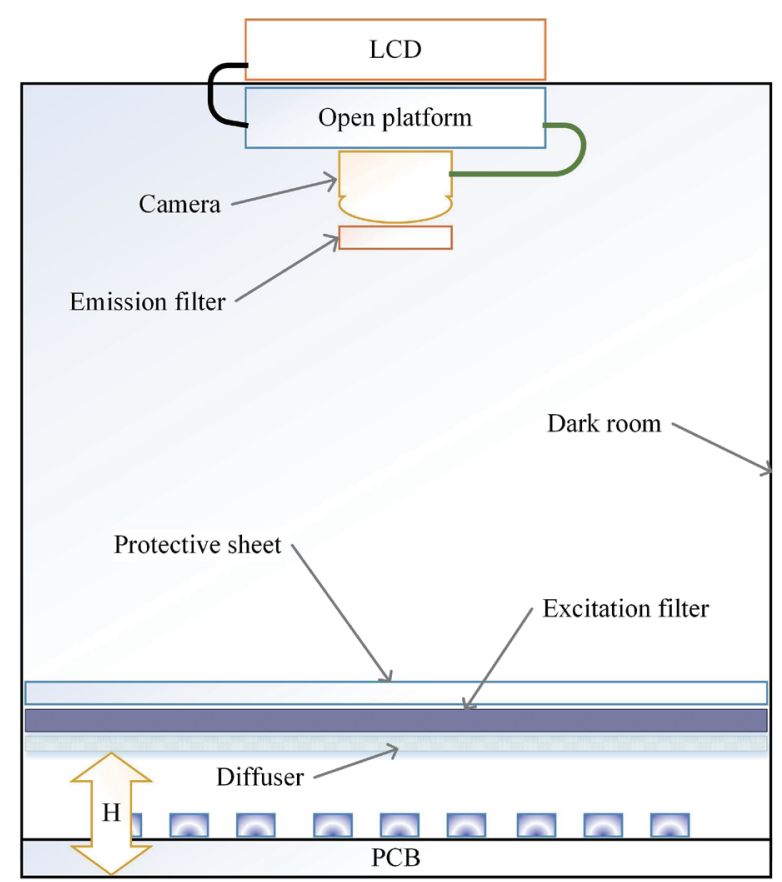

Fig. 1. (Color online) Block diagram of the proposed gel documentation system.

One of the recently actively developed single-board computers (SBCs) can be used as the open platform, as shown in Fig. 1. Typically, Raspberry Pi and its alternatives are a good choice. Most of them provide a wired and wireless network interface, so they can be connected to the network without any further components. A network file-sharing protocol can be used for a user's computer to seamlessly access the captured images on the SBC. As the liquid-crystal display (LCD) in the figure is also available at low cost for open platforms, there are plenty of choices. The LCD is usually connected to the open platform via a parallel or high-definition multimedia interface (HDMI) and delivers live video and still captures from the camera through the various graphical user interface (GUI) programs available as the open source. Therefore, after the electrophoresis gel is placed on a protective sheet and the dark room is closed, the live video of the gel is displayed on the LCD. When the shutter button on the GUI is pressed, the gel image is stored in the open platform and is immediately available to the user's computer. Cameras developed for smartphones are providing low-light photography suitable for the gel documentation system. Some of these cameras are available with a mobile industry processor interface (MIPI) or a USB interface, so their adoption into the gel documentation system can provide satisfactory performance. In this study, one of the Raspberry Pi SBCs was selected as an open platform, and the LCD and camera were chosen from among those developed for the platform. Selection criteria included cost and size as well as ease of development and management.

As shown in Fig. 1, the blue transilluminator for exciting gel fluorescence consisted of a LED board, a diffuser for uniform lighting, a blue excitation filter for improving the signal-to- 
noise ratio, and a protective film to protect it. On the LED board, $8 \times 91500$ mcd blue LEDs were arranged at $7 \mathrm{~mm}$ intervals. As the excitation and emission filter, 3-mm-thick deep blue (Acrylic \#2424) and amber acrylic (Acrylic \#2422) sheets were used. They increase the signalto-noise ratio by blocking light of around $500 \mathrm{~nm}$ where LED excitation and gel fluorescence wavelengths overlap. In order to make the system compact, the height indicated by the arrow ' $\mathrm{H}$ ' in Fig. 1 must be minimized as much as possible. The height from the protective sheet to the camera was not a design parameter because it was determined to fit the maximum size of gels in the field of view. In this work, we adopt the latest two diffusers considering price and availability and compared them with the existing one. The two new diffusers and the existing one are listed in Table 1

The images in Fig. 2 were for different distances between the 'Existing' diffuser and the LEDs. The image on the right was taken when the distance was large. That is, the image on the right was taken from a long distance. The LED patterns are visible in both cases, but appear blurry when the distance is large. We compared the uniformity of the diffusers with respect to the distance to select the diffuser. As the measure of uniformity, the difference between the maximum and minimum brightness or the relative standard deviation of brightness has been developed, but the characteristics of the periodic pattern can be clearly seen in the autocorrelation image. ${ }^{(20-24)}$ Since the LEDs are periodically arranged, if a partial image including one LED can be extracted, the ratio of the brightness of the brightest portion to that of the surrounding dark portion of the partial image can be obtained and used as a measure of uniformity. However, it is not easy to extract a partial image including only one LED, and it is more difficult if the diffuseness level is high, as shown in the right image of Fig. 2. Therefore,

Table 1

Diffusers tested.

\begin{tabular}{lccc}
\hline & Existing & New1 & New2 \\
\hline Product name & Inventables, 23114-02 & 3M, 3635-70 & LG, LDL90DF \\
Thickness of 1 sheet & $220 \mu \mathrm{m}$ & $80 \mu \mathrm{m}$ & $125 \mu \mathrm{m}$ \\
Total thickness & $220 \mu \mathrm{m}$ & $140 \mu \mathrm{m}$ & $230 \mu \mathrm{m}$ \\
\hline
\end{tabular}

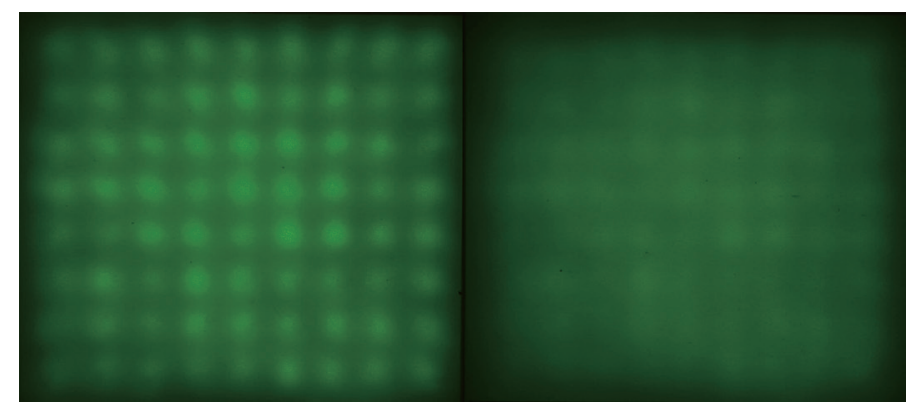

(a)

(b)

Fig. 2. (Color online) When the distance from the LED to the diffuser is (a) small (14 $\mathrm{mm})$ and (b) large (existing diffuser, $19 \mathrm{~mm}$ ). 
the autocorrelation image is analyzed in this paper instead of the image intensity itself. If the diffuser and the LEDs are farther apart, the periodic pattern disappears, as shown in the right autocorrelation image in Fig. 3. In contrast, when the diffuser is close to the LEDs and the diffusing performance worsens, the autocorrelation function will change more rapidly, as shown in the left image in Fig. 3. In the simulation of a freeform lens, the intensity distribution could be observed directly because there was no noise. ${ }^{(22)}$ However, to measure the uniformity in a real image, it is advantageous to observe the expectation or the power of intensity considering the effect of noise. The rate at which the brightness decreases from the peak to the periphery in the LED image can be used as a uniformity measure. ${ }^{(22)}$ Likewise, how quickly the autocorrelation decays from the center to the outside can be used as a measure of uniformity. Therefore, the radius of the center portion is calculated from the sharp autocorrelation image (left image in Fig. 3) of the LED pattern (left image in Fig. 2), and the average value along the boundary of the circle was obtained. The ratio of the average value to the center value is used as the uniformity measure. The radius of the center lobe was calculated from the average distance from the center to the nearest valleys. The radius was the average distance divided by the square root of 2. The ' + ' and 'o' symbols and the green circle in Fig. 4 show the autocorrelation image center, the nearest valleys, and the boundary of the obtained lobe, respectively. The uniformity measure $r$ is represented as

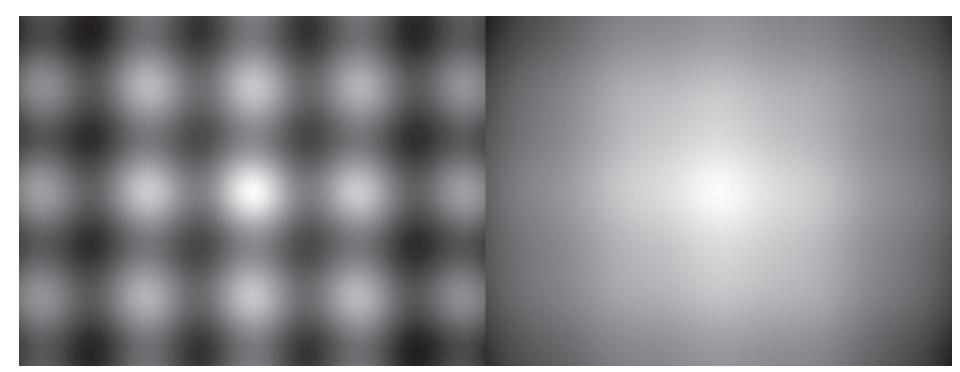

Fig. 3. Autocorrelation images of the images shown in Fig. 2.

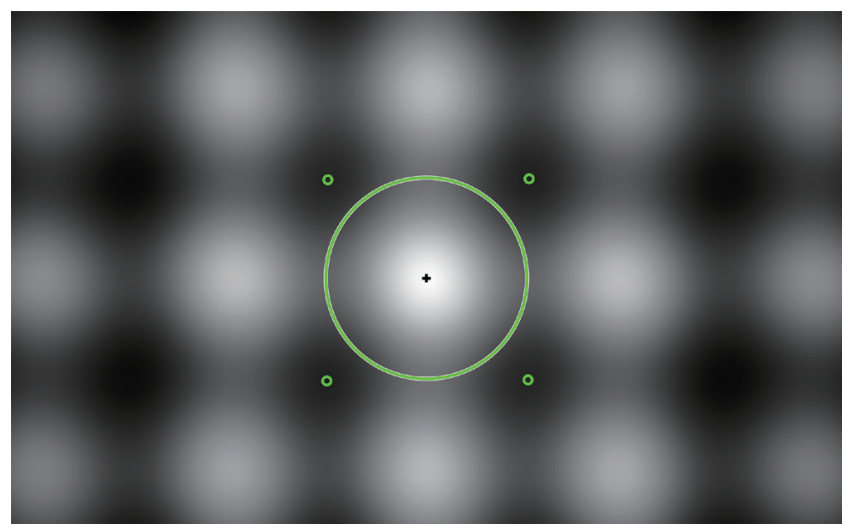

Fig. 4. (Color online) Determination of position for uniformity measurement. 


$$
r=\frac{\frac{1}{n} \sum_{C} R(i, j)}{R(0,0)},
$$

where $R(\bullet), n$, and $\sum(\bullet)$ are the autocorrelation function, the number of samples on the peripheral circle, and the sum of the autocorrelation values on the samples. The more uniform the image, the closer the uniformity $r$ will approach unity.

\section{Result}

For the open platform SBC, the camera, and the display, we chose Raspberry Pi $3 \mathrm{~B}+$, Raspberry Pi Camera Module V2 (Table 2), and a 5-inch HDMI LCD with $800 \times 640$ pixel resolution (Ntrex Co. Ltd., Korea), respectively. The SBC was connected to the Internet via WiFi, enabling the user's computer to access the SBC's file system using the server message block (SMB) protocol, which was one of the protocols for network file sharing. We used the 'PyCam' and 'wxWidgets' libraries to create a GUI with live view windows and shutter buttons, which automatically appeared on the LCD screen after booting. As described in the previous section, the user places an electrophoretic gel on a protective sheet, confirms it through the live view, and then presses the shutter button to take a gel image.

To make the thickness of the diffusers similar, 'New1' and 'New2' diffusers were each made thicker by attaching 2 sheets. As a result, 'Existing', 'New1', and 'New2' were 220, 140, and $230 \mu \mathrm{m}$ thick, respectively, as shown in the bottom row of Table 1. The new diffuser sheets adopted in this study showed similar performances at much lower heights than the existing ones. To compare their performances, LED light was photographed while changing the spacing between the diffuser and the LED printed circuit boards (PCBs). Because LED light was too bright, we assumed that the blue light exciting the fluorescence would scatter similarly regardless of wavelength, and only the long tail portion of the excitation blue light wavelength was photographed. To do this, an orange glass long-pass filter (\# 45064, Edmund Optics Inc., NJ, USA) with a slightly shorter cutoff wavelength was used instead of the emission filter.

The image on the left in Fig. 5 is taken with the 'Newl' diffuser $15.5 \mathrm{~mm}$ above the LED PCB and the image on the right was taken with the 'Existing' diffuser $32 \mathrm{~mm}$ above the LED PCB. The pattern of the LEDs was different between the two images, but it was unclear whether or not their uniformities were similar. Their autocorrelation images are shown in Fig. 6. The green circle in each image shows the sampling position of the surrounding autocorrelation

Table 2

Specifications of the Raspberry camera.

\begin{tabular}{lc}
\hline Feature & Description \\
\hline Product name & Camera module V2 \\
Manufacturer & Raspberry Pi \\
Video mode & $1080 \mathrm{p} 30,720 \mathrm{p} 60,640 \times 480 \mathrm{p} 60 / 90$ \\
Sensor resolution & $3280 \times 2464$ \\
\hline
\end{tabular}




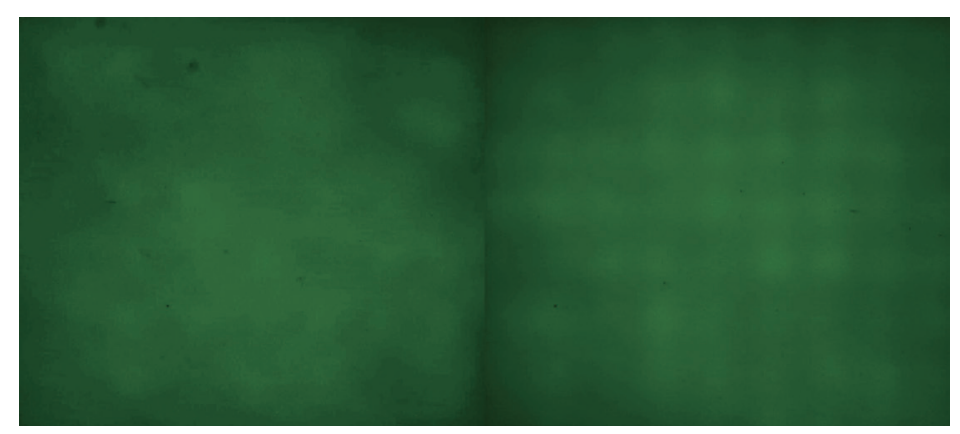

(a)

(b)

Fig. 5. (Color online) LED images with (a) 'New1' diffuser and (b) 'Existing' one.

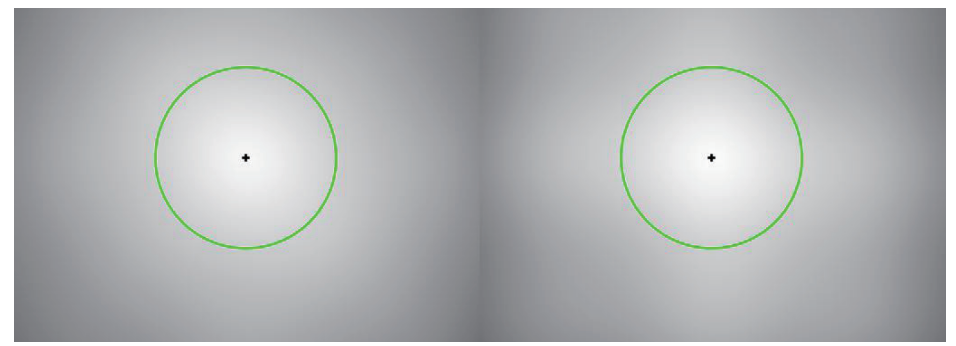

Fig. 6. (Color online) Autocorrelation images and the sampling positions of the images in Fig. 5.

for comparison with the center value in each image. The average autocorrelation along the circle was divided by the center autocorrelation (marked ' + ') to calculate the uniformity. Figure 7 shows the autocorrelation profiles along the green circles of the autocorrelation images. The figure shows that the patterns of change are different, but the average values are similar.

Figure 8 shows the uniformity of each diffuser against the distance between the LED PCB and the diffuser. The distance between the existing diffuser and the LED PCB and the uniformity were $32 \mathrm{~mm}$ and 0.90 , respectively. The uniformity of the existing diffuser was denoted by the vertical line in Fig. 2. The 'New2' diffuser exhibits a slightly lower performance than the existing one, but the 'Newl' diffuser is compatible with the existing diffuser from above $15.5 \mathrm{~mm}$. These results suggest that the adoption of a modern diffuser could reduce the height of the transilluminator to half or less.

Figure 9 shows gel images taken with 'Newl' (b) and 'Existing' diffuser (a). The images were taken after electrophoresis with ladder and DNA amplicons loaded on an agarose gel stained with ChamelGreen I nucleic acid gel stain (Dawinbio Inc., Korea). The first two and last columns from the left side of each gel image show the DNA ladder and the rest show DNA amplicons amplified sufficiently. Except for the mosaic pattern seen at the center of the image taken using the 'Existing' diffuser, no flaw like the LED pattern was seen in either image. The mosaic pattern was considered to be due to the liquid remaining on the gel. We concluded from the qualitative evaluation by our team and bioengineers that the quality of both images was the same. In other words, performance did not degrade when the height of the transilluminator was reduced to half. 


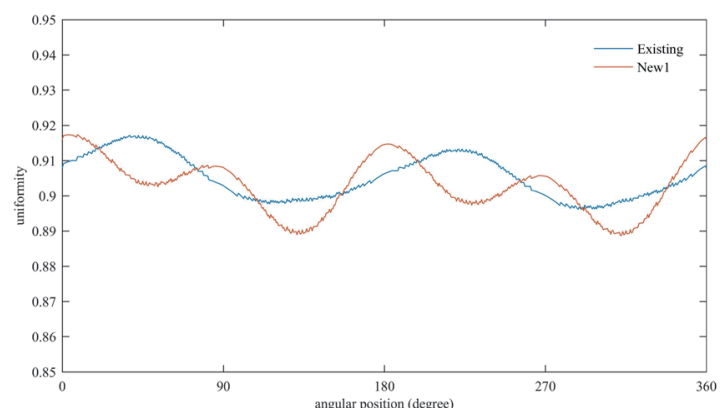

Fig. 7. (Color online) Profiles of autocorrelations along the green circles in Fig. 6.

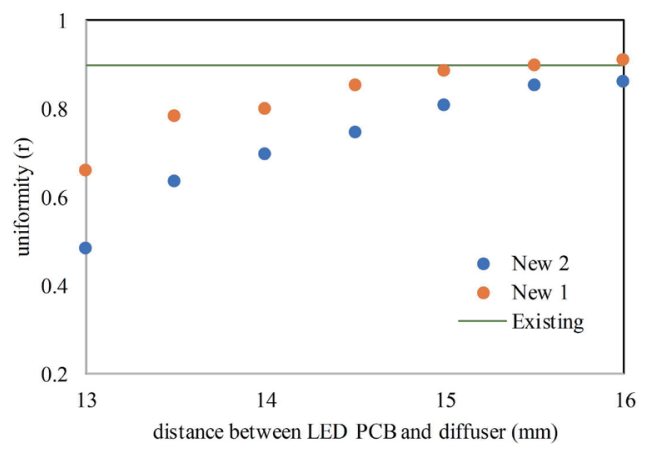

Fig. 8. (Color online) Uniformity versus distance between diffuser and LED.

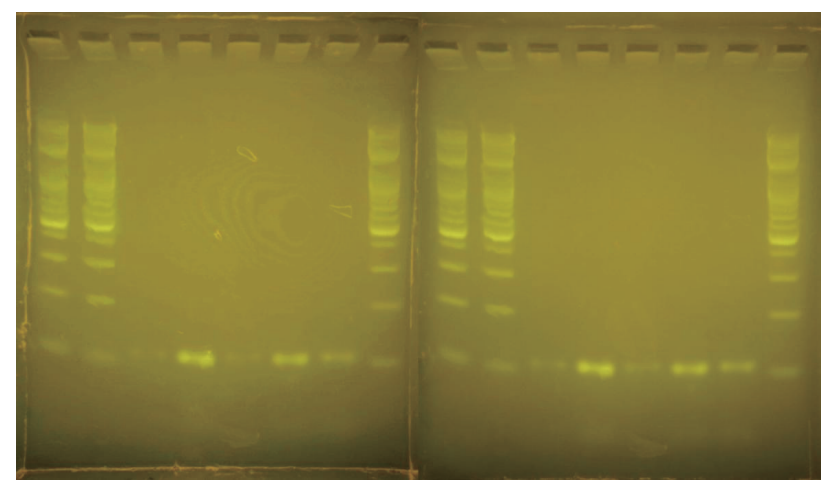

(a) (b)

Fig. 9. (Color online) Gel images taken with (a) 'Existing' diffuser and (b) 'Newl' diffuser.

\section{Conclusions}

In this paper, the IoT-type gel document system was proposed. In the IoT format, the user interface is via the network, so the system only needs to perform the original detection task. Therefore, developing a system in this form will provide a compact and inexpensive system. We proposed systems that match the new trends, applying open platforms, cameras for open platforms, and new optical diffusers. We also suggested a way to compare the performance of the new diffusers with the existing diffuser. As a result, the height of the transilluminator was reduced to less than half when using a new diffuser.

\section{Acknowledgments}

This research was supported by the Basic Science Research Program through the National Research Foundation of Korea (NRF) funded by the Ministry of Science, ICT \& Future Planning (NRF-2017R1A2B4002679) and by The Leading Human Resource Training Program of Regional Neo-industry through the National Research Foundation of Korea (NRF) funded by the Ministry of Science, ICT \& Future Planning (NRF-2016H1D5A1909654). 


\section{References}

1 S. Singh, K. Kumari, S. Chaturvedi, N. Pandey, and A. Varghese: Applications of Biotechnology for Sustainable Development (Springer, Singapore, 2017) p. 7.

2 A. K. Rai, S. Sanjukta, R. Chourasia, I. Bhat, P. K. Bhardwaj, and D. Sahoo: Bioresour. Technol. 235 (2017) 358.

3 N. Kumar, D. Shikha, S. Kumari, B. K. Choudhary, L. Kumar, and I. S. Singh: Appl. Biochem. Biotechnol. 185 (2018) 34.

4 X. Zhu, H. Shi, Y. Shen, B. Zhang, J. Zhao, and G. Li: Nano Res. 8 (2015) 2714.

5 J. A. Meyers, D. Sanchez, L. P. Elwell, and S. Falkow: J. Bacteriol. 127 (1976) 1529

6 J.-C. Ogier, O. Son, A. Gruss, P. Tailliez, and A. Delacroix-Buchet: Appl. Environ. Microbiol. 68 (2002) 3691.

7 A. Rotaru, S. Dutta, E. Jentzsch, K. Gothelf, and A. Mokhir: Angew. Chem. Int. Ed. 49 (2010) 5665.

8 S. H. Yi, L. C. Xu, K. Mei, R. Z. Yang, and D. X. Huang: Forensic Sci. Int. Genet. 11 (2014) 117.

9 W. Chen, G. J. Maghzal, A. Ayer, C. Suarna, L. L. Dunn, and R. Stocker: Free Radical Biol. Med. 115 (2018) 156.

10 A. J. Paredes, T. Naranjo-Palma, H. M. Alfaro-Valdés, A. Barriga, J. Babul, and C. A. Wilson: Anal. Biochem. 517 (2017) 31.

11 F. J. Alba, A. Bermúdez, and J. R. Daban: Electrophoresis 22 (2001) 399.

12 T. Ohta, S.-I. Tokishita, and H. Yamagata: Mutat. Res. Genet. Toxicol. Environ. Mutagen. 492 (2001) 91.

13 C. F. Brunk and L. Simpson: Anal. Biochem. 82 (1977) 455.

14 M. M. Becker and Z. Wang: J. Mol. Biol. 210 (1989) 429.

15 T. Goldmann, A. Zyzik, S. Loeschke, W. Lindsay, and E. Vollmer: J. Biochem. Biophys. Methods 50 (2001) 91.

16 J. Gubbi, R. Buyya, S. Marusic, and M. Palaniswami: Future Gener. Comput. Syst. 29 (2013) 1645.

17 A. F. A. Rahman, M. Daud, and M. Z. Mohamad: Proc. Int. Conf. Internet of Things and Cloud Computing (2016) 79.

18 A. Botta, W. De Donato, V. Persico, and A. Pescapé: Future Gener. Comput. Syst. 56 (2016) 684.

19 W. Huang, F. Fang, X. Ma, Z. Dong, and X. Xu: IEEE 10th Conf. Industrial Electronics and Applications (IEEE, 2015) 2030

20 H. Jian, H.-L. Ke, Y. Wang, L. Jing, H. Liu, Q. Sun, and Z.-Q. Wang: Optik - Int. J. Light Electron Opt. 134 (2017) 128.

21 D. Ma, Z. Feng, and R. Liang: Appl. Opt. 54 (2015) 498.

22 Z. Zhao, H. Zhang, H. Zheng, and S. Liu: Opt. Commun. 410 (2018) 123.

23 Z. Zhu, D. Ma, Q. Hu, Y. Tang, and R. Liang: Opt. Express 26 (2018) A54.

24 K. Wang, D. Wu, Z. Qin, F. Chen, X. Luo, and S. Liu: Opt. Express 19 (2011) A830. 